DOI: 10.12957/demetra.2018.33112

\title{
O excesso de peso na perspectiva de gestantes assistidas na atenção primária à saúde de um município da região do Norte Fluminense
}

\section{Overweight in the perspective of pregnant women assisted in the primary health care of a municipality of the North Fluminense region}

\author{
Carolina da Costa Pires ${ }^{1}$ \\ Mariana Leal Rodrigues ${ }^{2}$ \\ Jane de Carlos Santana Capelli ${ }^{3}$ \\ Marta Maria Antonieta de Souza Santos ${ }^{4}$ \\ Mirian Ribeiro Baião? \\ 1 Universidade Federal do Rio de Janeiro, \\ Faculdade de Medicina, Instituto de Atenção à \\ Saúde São Francisco de Assis. Rio de Janeiro, \\ RJ, Brasil. \\ 2 Universidade Federal do Estado do Rio de \\ Janeiro (Unirio), Departamento de Saúde \\ Coletiva. Rio de Janeiro, RJ, Brasil. \\ ${ }^{3}$ Universidade Federal do Rio de Janeiro, Campus \\ UFRJ-Macaé Professor Aloisio Teixeira. Macaé, \\ RJ, Brasil. \\ ${ }^{4}$ Universidade Federal do Rio de Janeiro, \\ Instituto de Nutrição Josué de Castro. Rio de \\ Janeiro, RJ, Brasil. \\ Correspondência / Correspondence \\ Carolina da Costa Pires \\ E-mails: c_pires4@hotmail.com;dac@ \\ hesfa.ufri.br
}

\section{Resumo}

Este trabalho tem como objetivo investigar o excesso de peso na gestação na perspectiva de mulheres adultas usuárias da Atenção Primária à Saúde (APS) do município de MacaéRJ. Trata-se de pesquisa de abordagem qualitativa, do tipo descritiva, fundamentada na perspectiva teórica interpretativista. Realizaram-se entrevistas em profundidade, cujos dados foram submetidos à análise de conteúdo temática adaptada de Bardin. Foram entrevistadas 12 gestantes, maiores de 20 anos, com diagnóstico nutricional de excesso de peso, residentes em Macaé-RJ e que realizavam pré-natal em duas unidades da APS do município, em 2017. O gestar com excesso de peso foi um desafio para as entrevistadas por suscitar percepções e sensações ambíguas de naturalização do peso excessivo, baixa autoestima, limitação na realização de atividades cotidianas e temor em relação ao que esperar do próprio corpo no puerpério. Foi possível apreender que os sentidos atribuídos ao gestar com excesso de peso demandam humanização, integralidade e efetividade da assistência nutricional, para que seja compromissada com a saúde das mulheres, suas histórias de vida e relações sociais às quais estão submetidas, a fim de minimizar a problemática do excesso de peso gestacional e suas consequências em gestantes adultas assistidas pela APS do município de Macaé-RJ.

Palavras-chave: Gestante. Excesso de Peso. Imagem Corporal. Pesquisa Qualitativa. 


\section{Abstract}

This study aims to investigate overweight during pregnancy from the perspective of adult women attending Primary Health Care (PHC) in the city of Macaé-RJ, Brazil. It is a research of qualitative approach and descriptive type, based on the theoretical interpretative perspective. We conducted in depth interviews, whose data were submitted to the analysis of thematic content adapted from Bardin. A total of 12 pregnant women, over 20 years of age, with a nutritional diagnosis of overweight residing in Macaé-RJ, who underwent prenatal care in two PHC units in the municipality in 2017, were interviewed. Overweight was a challenge for those interviewed for raising perceptions and ambiguous sensations of naturalization of overweight, low selfesteem, limitation in performing daily activities and fear of what to expect from the body itself in the puerperium. It was possible to understand that the senses attributed to overweight management require humanization, integrality and effectiveness of nutritional assistance, so that it is committed to the health of women, their life histories and social relations to which they are subjected, in order to minimize problems of gestational overweight and its consequences in adult pregnant women assisted by PHC in the city of Macaé-RJ.

Keywords: Pregnant Woman. Overweight. Body Image. Qualitative Research.

\section{Introdução}

O processo de gestar uma nova vida desencadeia inúmeras alterações metabólicas e fisiológicas que implicam aumento das necessidades nutricionais e energéticas da mulher, demandando a adoção de alimentação adequada e saudável. A prevenção do ganho excessivo de peso na gestação e da retenção de peso no pós-parto é aspecto fundamental na atenção pré-natal, por estes serem fatores de risco para o desenvolvimento da obesidade em mulheres. ${ }^{1-3}$

A World Health Organization ${ }^{4}$ define o sobrepeso e a obesidade como um acúmulo anormal ou excessivo de gordura que pode comprometer a saúde. Atualmente, mais da metade da população feminina brasileira acima de 18 anos está com excesso de peso, sendo 18,9\% obesos. ${ }^{5}$

O fenômeno da obesidade assume diversos sentidos, modulados por valores socioculturais e simbólicos presentes nos diferentes contextos sócio-históricos. ${ }^{6}$ Em geral, em meio à pobreza, o excesso de peso costuma ser valorizado pelas mulheres, pois se desvincula da ideia de fraqueza, 
de doenças e privação alimentar. Nesse contexto, o corpo obeso representa força e suficiência alimentar, ainda que paradoxalmente associado ao surgimento de sintomas como "dor nas pernas", "dor na coluna", "falta de ar" e "menor disposição", limitantes das atividades cotidianas e laborais.

Nas últimas décadas, a ressignificação social do corpo grávido por meio da exposição da "barriga", antes considerada um tabu comportamental, e da afirmação da maternidade como uma autorrealização e liberdade de escolha contribuíram para amenizar mudanças na percepção da autoimagem corporal da mulher, que podem resultar das transformações físicas, hormonais e psicológicas geradas pela gravidez. ${ }^{8}$

Embora o lugar e a valorização da maternidade no âmbito sociocultural tenham se modificado e variado ao longo do tempo, por influência de diferentes contextos sócio-históricos e culturais, o devotamento e o sacrifício voluntário ainda se fazem presentes no ideário social como pilares da construção de uma nova identidade: a mulher-mãe. ${ }^{9}$

Estudo envolvendo a satisfação com a imagem corporal entre mulheres grávidas e não grávidas concluiu que, na gravidez, o nível de satisfação corporal foi significativamente maior. ${ }^{10}$ Esse achado aponta para o fato de que, em geral, durante a gestação, a mulher tende a ficar despreocupada com o ganho de peso, por compreender que se trata de uma condição natural do período gestacional. Por sua vez, a equipe de saúde precisa olhar as gestantes considerando sua perspectiva, pois estas orientam as práticas de cuidar inovadoras direcionadas à singularidade e à particularidade da experiência por elas vivida. Somente com essa perspectiva, a equipe de saúde pode desenvolver, junto às gestantes, ações específicas com uma abordagem que extrapole a dimensão biológica e contemple os aspectos psíquicos, sociais e afetivos, sobretudo quando uma condição de risco se torna aparente.

A construção de vínculo, o diálogo franco e aberto entre o profissional de saúde e a gestante, o diagnóstico nutricional precoce e a eficácia do protocolo terapêutico de controle de ganho de peso durante a assistência pré-natal podem contribuir para que a gestante compreenda o impacto do excesso de peso para sua saúde e da criança. ${ }^{11}$

Neste sentido, o presente estudo tem por objetivo investigar o excesso de peso no período gestacional, na perspectiva de mulheres adultas, usuárias da atenção primária à saúde do município de Macaé-RJ.

\section{Metodologia}

Realizou-se pesquisa de abordagem qualitativa, do tipo descritiva, fundamentada na perspectiva teórica interpretativista, sendo utilizada a análise de conteúdo temática adaptada de Bardin ${ }^{12}$ como abordagem metodológica. 
O estudo foi desenvolvido em duas unidades de saúde do município de Macaé: Estratégia de Saúde da Família (ESF) Cajueiros e Núcleo de Apoio à Mulher e à Criança - NUAMC Visconde, entre os meses de fevereiro e agosto de 2017.

O município de Macaé, que passou de "Princesinha do Attântico" a "Capital Nacional do Petróleo", situa-se na região nordeste do estado do Rio de Janeiro, a 180 quilômetros da capital fluminense, e conta com uma população estimada de 244 mil habitantes. ${ }^{13}$ A presença de indústrias na região e a ocupação desordenada ocasionaram mudanças importantes no sistema alimentar. A cultura da pesca e a agricultura de subsistência sofreram com o avanço da industrialização e exploração do petróleo na região, afetando as práticas alimentares, o que pode ter contribuído para o aumento do sobrepeso e da obesidade na população macaense, inclusive em gestantes. ${ }^{14,15}$

Para o desenvolvimento do estudo, realizou-se contato com as gestoras das unidades de saúde para apresentação da pesquisa, com posterior aprovação e organização de um cronograma de atividades. Inicialmente, por acesso aos registros em prontuário institucional, foram identificadas as gestantes que preenchiam os critérios de inclusão: residir no município de Macaé; ser adulta (maior de 20 anos) e apresentar diagnóstico de excesso de peso segundo o IMC por semana gestacional (sobrepeso ou obesidade). Dos prontuários, também foram extraídas informações para caracterização das participantes do estudo.

Em seguida, foi realizado contato diretamente com as gestantes nos dias das consultas de prénatal ou por intermédio dos agentes de saúde. Quando do encontro com a pesquisadora principal, as gestantes receberam explicações sobre a pesquisa, e as que aceitaram participar assinaram o termo de consentimento livre e esclarecido e foram encaminhadas a uma sala reservada, para que se garantisse a privacidade da entrevistada.

Empregou-se o critério de saturação das falas para delimitação do número de participantes a compor o grupo a ser investigado, ${ }^{16}$ totalizando 12 gestantes adultas com diagnóstico de excesso de peso.

Na construção dos dados foi utilizada entrevista semiestruturada, gravada digitalmente e transcrita na íntegra, para garantir o registro das falas, dos códigos verbais e não verbais, como de entonações de voz, interjeições e silêncios. Apesar de o roteiro da entrevista ter abordado os temas "a história da gravidez", "o gestar e o excesso de peso", "o pré-natal e cuidado nutricional", no presente manuscrito, apenas serão apresentados os sentidos do gestar com excesso de peso.

O estudo foi aprovado pelo Comitê de Ética em Pesquisa da Escola de Enfermagem Anna Nery (CEP EEAN) da instituição proponente (UFRJ) e da instituição coparticipante (Secretaria Municipal de Saúde de Macaé), conforme prevê a Seção I da Resolução no 466/2012 do Conselho Nacional de Saúde, que estabelece as diretrizes e normas para pesquisas envolvendo seres humanos. ${ }^{17}$ 


\section{Resultados e Discussão}

\section{Caracterização dos sujeitos do estudo}

Foram entrevistadas 12 gestantes, das quais quatro eram naturais do município de Macaé, três do município do Rio de Janeiro e as demais naturais de outros municípios do estado do Rio de Janeiro (duas), da Bahia (duas) e de Minas Gerais (uma). Oito eram multíparas e quatro, primíparas. Uma havia completado o ensino fundamental; cinco, o ensino médio; outras cinco não concluíram esse segmento e apenas uma cursava o nível superior. Oito eram do lar, uma estudante e três exerciam trabalho remunerado. À época da entrevista, todas viviam com seus companheiros, que, na maior parte dos casos, garantiam o orçamento da família.

Quanto à adesão ao calendário mínimo de seis consultas pré-natais, ${ }^{1}$ somente quatro das entrevistadas compareceram a seis ou mais consultas, enquanto oito referiram comparecimento a menos de seis. O diagnóstico nutricional apontou que metade das entrevistadas apresentava sobrepeso e outra metade, obesidade.

\section{O tema e suas categorias}

O gestar com excesso de peso foi apreendido a partir das falas das entrevistadas por meio das seguintes categorias temáticas: corpo e imagem corporal; autoestima; limitações físicas; e expectativas no pós-parto.

\section{Corpo e imagem corporal}

A condição de gerar uma vida impõe mudanças físicas extremamente intensas, capazes de gerar conflitos íntimos identitários a partir das novas conformações corporais adquiridas, sobretudo quando o corpo exerce o papel de interlocutor e autoafirmação social. ${ }^{18}$

Para Le Breton: ${ }^{19}$

[...] o corpo é colocado não como algo indistinto do homem, mas como uma posse, um atributo, um outro, um alter ego. O homem é a fantasia desse discurso, o sujeito suposto. A apologia ao corpo é, sem que tenha consciência, profundamente dualista, opõe o indivíduo ao corpo e, de maneira abstrata, supõe uma existência para o corpo que poderia ser analisada fora do homem concreto (p. 10).

A busca pelo corpo magro está relacionada a um padrão de beleza e saúde amplamente difundido em meios de comunicação na sociedade contemporânea. Uma silhueta definida e musculosa representa um objetivo na vida de muitos indivíduos, particularmente do gênero 
feminino. Ser magro exprime o espírito de determinação, reflete a conquista do sucesso e é um indicativo de juventude, saúde e sexualidade. ${ }^{20}$

Serra \& Santos $^{21}$ afirmam que os meios de comunicação verbalizam os propósitos do mundo empresarial no que se refere ao estímulo da compra e venda de bens e serviços que proporcionem o alcance da saúde e do corpo ideal. No mundo globalizado, campanhas publicitárias, programas de televisão, mídia impressa e digital constituem veículos de informações que confrontam o saber científico com as representações e expectativas criadas ao redor da obtenção do corpo considerado perfeito.

Nas falas de algumas das entrevistadas, foi possível identificar a influência dos discursos midiáticos sobre a forma corporal socialmente idealizada e sobre os significados atribuídos à sua conquista, que remetem a características individuais em voga nos dias atuais, como o espírito de vencedor e a busca pela perfeição. Todavia, mesmo um pouco frustradas pela percepção das mudanças corporais decorrentes do período gravídico, a maturidade conferiu a essas mulheres resiliência em relação à abdicação temporária do alcance do corpo ideal e a certeza de que o bem-estar psicológico promoverá a vivência plena da gestação, do parto e da maternidade e, em momento oportuno, poderão reconquistar as formas corporais:

Mudou um bocado [...] porque eu tava com músculo já, eu tava definida. Aí, depois eu parei de praticar a musculação, aí, você ganha gordura e perde massa magra, né? [...] Eu tinha pavor de imaginar que eu poderia ter gordura no lugar de massa magra [...] Então, mudou um pouquinho. Agora, meus musculozinhos não têm mais [...] Eu sempre fui muito obcecada [...] nada me derrubou nunca. Eu sempre queria mais e mais a respeito de corpo. Só que, agora, eu tô mais madura e enfiei um negócio na minha cabeça: tudo isso é psicológico. Nada é pra sempre. Então, tudo tem o seu tempo. Eu tive tempo de ficar malhando, agora, eu tô grávida (GA09).

Apesar de constituírem a maior reserva energética do organismo, participarem dos processos digestórios e serem essenciais para a manutenção da temperatura corpórea e produção de hormônios vitais, as células gordurosas (adipócitos) contrapõem os arquétipos estéticos atuais e ganham uma conotação pejorativa. Embora mais da metade das mulheres brasileiras apresente o diagnóstico de sobrepeso e obesidade, estar acima do peso significa ser preterido socialmente, uma vez que o excesso de peso já é reconhecido como uma doença.,

Em estudo conduzido por Clark et al., ${ }^{22}$ o novo propósito de vida advindo da gestação motivou mulheres a lidarem de maneira mais positiva frente às alterações corporais, uma vez que o corpo deixa de assumir papel meramente estético para atuar como uma unidade funcional reprodutiva, o que também foi possível para as gestantes deste estudo:

Eu prefiro não comentar [...] [risos]. Vai ter uma faixa que eu vou ter que usar, um sutiã pra botar o peito no lugar. Vou ter que assumir. Fazer o quê?? Foi uma escolha minha, eu que quis. Foi por uma boa causa [...] foi por causa da minha filha, foi por amor. Vai valer à pena! (GLO3). 
[...] Ter energia pra cuidar, é o que eu mais tenho na cabeça. Eu não quero ficar uma pessoa, uma mulher sem energia pra quando a minha filha correr, eu não poder correr atrás dela. Porque você sabe que criança [...] você precisa de gás e de energia (GC08).

Para Le Breton, ${ }^{19}$ o corpo separa o indivíduo dos outros e do mundo, conferindo-lhe a diferenciação individual. Contudo, para o imaginário da modernidade, não deve ser tratado como um elemento de exclusão, mas de inclusão, constituindo um elo com os sistemas simbólicos dos membros da comunidade.

A aparência e a postura não são adquiridas, mas construídas pela mulher, dependem do meio cultural a que estão agregadas. Cuidar do corpo significa ouvi-lo constantemente tanto para buscar boa saúde como para identificar o que está errado. O corpo tem o poder de ajudar a manter a boa aparência do sujeito e, por consequência, a sua satisfação. ${ }^{23}$ (p. 218).

Vale ressaltar que aos corpos feminino e masculino não são somente atribuídas características fisiológicas, mas significados a partir de processos de construção social. É no corpo que se inscrevem regras familiares, de classe e de gênero. O corpo é o suporte de construção identitária realizada pela estrutura social sobre a pessoa. É o mediador prático entre o simbólico e o social, e sustenta as estruturas da sociedade de maneira particularmente intensa e dolorosa. É por meio das dores e prazeres individuais que os comportamentos individuais e sociais são percebidos; o corpo é, assim, um locus privilegiado da cultura. Compreender de que maneira as gestantes experimentam a gravidez com sobrepeso e obesidade é ir além das subjetividades e escolhas individuais e se aproximar de um fenômeno ainda mais amplo da condição feminina na sociedade brasileira, na qual as mulheres são submetidas a inúmeras formas de violência física e simbólica. ${ }^{24}$

No relato de duas entrevistadas, foi possível observar inquietação com alterações corporais que podem se tornar marcas definitivas após a gestação. Em obesas ou com história familiar de obesidade, a tensão foi ainda maior, pois a ideia de irreversibilidade dessa condição despertou o sentido permanente de depreciação e inconformidade, oriundos do estigma social em torno da gordura, ${ }^{25}$ o que pode ser verificado pelo uso das palavras "preocupação", "acabada", "medo" e "engordar muito".

Eu tenho preocupação [...] porque as pessoas falam muito dessa questão de gravidez, que a mulher depois que engravida fica acabada, né? [...] Eu sou acima do peso (GC08).

Ai, eu tô com medo [...] por causa da minha mãe, eu tenho tendência de engordar [risos nervosos]. Mas vamos ver, se eu vou engordar muito [...] (GDO2). 
Hiatos na construção da imagem corporal favorecem a busca de padrões e ideais muitas vezes incompatíveis com a realidade de algumas mulheres e, até mesmo, artificiais. ${ }^{19}$ Segundo Kanno et al.:20

[...] a imagem corporal é a construção cognitiva e afetiva que o indivíduo realiza a respeito de seu próprio corpo. Esta imagem encontra-se estritamente relacionada ao ideal corporal que circula pela mídia na sociedade. Assim, as pessoas aprendem a avaliar seus corpos a partir da interação com o ambiente, sendo, portanto, a autoimagem desenvolvida e reavaliada continuamente. (p. 425).

Numa tentativa de superar o sentimento de fracasso e insatisfação pessoal, o corpo físico, mental e social trava inúmeras batalhas dentro e fora de si para absorver os diferentes saberes e práticas do seu grupo. ${ }^{19-21}$

Assim que acabar o resguardo, eu vou começar o "Mamãe Sarada", já ouviu falar? É um projeto que você faz, em 14 minutos, todos os exercícios voltados para aquilo que está no pós-parto. E se der um problema, e você sentir aquela flacidez na barriga, tem um exercício próprio que enrijece a barriga e volta o músculo pro lugar. É tudo voltado para pós-parto. É isso. Aí, primeiro, eu vou começar a fazer isso, porque não tem ninguém que possa fazer [...] 14 minutos por dia! A criança pode tá dormindo e você fazer [...] (GA09).

O ideal do "corpo sarado" revela um aspecto perverso da condição feminina experimentada pelas entrevistadas. Todas eram financeiramente dependentes do marido, donas de casa, e não exerciam profissão no momento da pesquisa. Não se sentindo inscrita no padrão de corpo feminino socialmente imposto como sendo desejável pelo homem, a mulher se considera "acabada"; isto é, vê seus ideais de feminilidade sendo postos em xeque. Daí a necessidade de buscar o corpo fetichizado ser mais premente no discurso do que a de exercer atividade remunerada e construir autonomia financeira. A expressão dessa preocupação pelas gestantes entrevistadas é um exemplo claro da reificação do corpo feminino e da consequente violência simbólica infligida pela dominação masculina.

\section{Autoestima}

Araújo et al. ${ }^{26}$ observaram que a maternidade justifica o aumento do peso corporal na gestação, uma vez que o excesso de peso, sendo positivamente recebido pela sociedade, autoriza à mulher esse "direito", em benefício do gestar e do cuidado da família.

Para duas gestantes, a responsabilidade com o lar e os filhos, o suporte marital e as limitações financeiras contribuíram para o desenvolvimento da resignação em relação ao próprio corpo, apesar de perceberem as modificações decorrentes do ganho de peso excessivo durante a gestação: 
Em si, a gente se acomoda ao fato da gente ser casada, de ter marido, sou mãe [...] realmente, ser mãe de três, não tem como você se cuidar. Ainda mais, na parte financeira mesmo, que não tem condições pra poder malhar. Até porque, as crianças estudam, eu sou dona do lar, né, cuido da casa e tudo mais [...] na verdade, a gente percebe a diferença. Mas, como eu sou muito bem casada, meu marido não me deixa pensar [...] só consigo saber o meu peso, a diferença do meu peso, só quando eu subo na balança [...] mas, isso não me afeta, a mim, diretamente, não me afeta (GI01).

[...] Hoje em dia, meu marido não critica uma estria, não critica nada, graças a Deus! [...] Só o meu peito que eu acho que está bem caído [risos]. Mas, tá tranquilo [...] (GLO3).

Machado, Vinholes \& Feldens ${ }^{27}$ consideram a autoestima materna um importante pilar na construção do vínculo entre mãe e filho. Por outro lado, Hauff \& Demerath ${ }^{28}$ verificaram que a falta de conforto e confiança no próprio corpo, decorrente de um IMC elevado, reduziram o tempo de prática de aleitamento materno pelas lactantes. Com base nessas observações, a avaliação da autoestima da gestante deveria ser um elemento considerado durante as consultas de pré-natal, de forma a auxiliá-la a lidar com os desafios impostos pela gestação.

Schultheisz \& Aprile ${ }^{29}$ entendem por autoestima "a valoração intrínseca que o indivíduo faz de si mesmo em diferentes situações e eventos da vida a partir de um determinado conjunto de valores eleitos por ele como positivos ou negativos" (p. 36). As alterações de peso, forma e tamanho corporal, características do período gravídico, podem desencadear sentimento de culpa e depreciação da autoestima, ${ }^{30}$ particularmente em mulheres com diagnóstico nutricional de excesso de peso e elevado ganho de peso gestacional:

Muda tudo, né? A gente fica se sentindo horrível (GD11).

Eu tenho preocupação sim, de como vai ficar o meu corpo [...] é horrível [...] (GC08).

A depreciação da autoestima causada pelo gestar com excesso de peso reflete sentimentos contraditórios gerados na construção social da gravidez, uma vez que o sacrifício do corpo feminino se traduz em mudanças difíceis de serem acolhidas. ${ }^{31}$

Apesar de a Portaria de n 569, de $1^{\circ}$ de junho de 2000 (Programa de Humanização no PréNatal e Nascimento) enaltecer a inclusão de ações protocolares em saúde que priorizem o bemestar físico e emocional da gestante, ${ }^{32} \mathrm{o}$ atendimento psicológico é destinado apenas às mulheres vítimas de violência (doméstica, sexual, psicológica, etc.) e em risco de depressão pós-parto, ${ }^{1}$ desconsiderando que no período gravídico possam surgir outros agravos que comprometam a saúde mental e a vivência da maternidade. ${ }^{33}$

Sui, Turnbull \& Dodd ${ }^{34}$ identificaram variações nos níveis de autoestima entre gestantes de acordo com a paridade. Multíparas apresentavam maior insatisfação corporal que primíparas, e a 
justificativa seria o maior ganho de peso gestacional e IMC. ${ }^{25,35}$ No presente estudo, as gestantes multíparas também expressaram maior insatisfação com o corpo:

[... É a terceira, então, tô aí na casa dos trinta e poucos quilos [...] Pior do que tá? [...] [multípara] (GI01).

Não tá me prejudicando em nada. Tô bem, graças a Deus! É, porque eu não engordei muito, de ficar toda feia. Tô me sentindo bem [primípara] (GDO2).

[...] Eu cheguei aos seis meses e uso as mesmas roupas que eu usava. Só usava roupa colada e continuo [primípara] (GCO7).

A visão que possuem de seu próprio corpo como repulsivo revela a adesão a uma imagem desvalorizada da mulher. Essa adesão à perspectiva masculina está longe de ser um ato consciente de um sujeito isolado, senso resultante de um poder, "inscrito duradouramente no corpo dos dominados sob forma de esquemas de percepções e de disposições (a admirar, respeitar, amar, etc.) que o tornam sensível a certas manifestações simbólicas do poder" ${ }^{24}$ (p. 63). E é na contradição entre o que é aceitável e adequado para a mulher-mãe e o que é esperado da mulher-esposa, que surgem as angústias das gestantes com sobrepeso, reforçando ainda mais o sentimento de baixa autoestima e minando a capacidade de autocuidado, em um período tão delicado da vida dessas mulheres.

\section{Limitacões físicas e expectativas no pós-parto}

Em virtude do aumento do peso materno e maior desenvolvimento fetal-entre o segundo e o terceiro trimestre da gestação, as mudanças corporais são mais intensas. Também é nesse período que ocorrem mais queixas físicas, já que as transformações do corpo limitam a realização de atividades cotidianas; por isso, devem ser abordadas e monitoradas durante a assistência prénatal e puerpério. ${ }^{33,36}$

Neste estudo, todas as entrevistadas se encontravam no período gravídico, e foram identificadas três referências aos agravos à saúde e incômodos relacionados ao ganho de peso excessivo e alteração das formas corporais:

[...] Varizes, eu não tinha varizes. Tô cheia de varizes, as pernas cheias de varizes. O corpo começou a inchar, as pernas incham muito. Não consigo andar [...] a pressão, coisa que eu não tinha, problema de pressão: tenho. Fiquei acima do peso [20Kg], muita coisa! (GA06).

Na agilidade de fazer as coisas [...] limita muito, né? [...] a gente não consegue mais fazer as coisas que fazia antes [...] eu não consigo mais vestir um sapato sem precisar de ajuda [...] caem as coisas no chão, eu deixo, esperando chegar pra pegar pra mim. Não tem como abaixar mais, né? Limita muito (GD11).

Estou inchada, com o quadril largo [...] (GT12). 
No relato abaixo, foi possível observar a aceitação do ganho de peso excessivo. De acordo com Meireles et al., ${ }^{37}$ o período gravídico promove um estado de tolerância ao aumento da gordura corporal por algumas mulheres, expresso pelo uso dos termos "bom", "tranquilo" e "desencanada".

Eu comecei com $74 \mathrm{Kg}$, já estava acima, mas eu creio que eu só engordei $10 \mathrm{~kg}$, e acho que na minha gestação está bom, devido ao tempo, né? Tá tranquilo [...] esse lance aí, eu tô desencanada (GLO3).

No entanto, quando inquiridas sobre as expectativas corporais no período pós-gravídico, verificou-se preocupação quanto à retomada aos padrões estéticos e a idealização de um corpo magro, sem marcas derivadas da gravidez:

Eu quero que volte como antes. Eu sei que vou ter que fazer uns esforços [...] eu gosto muito de caminhar, da academia, essas coisas eu gosto. Eu sei que vai ficar [...] não vai ser como era. Mas nada que eu não possa correr atrás do prejuízo e voltar como era antes (GA06).

O medo maior é só das estrias. O resto, a gente corre atrás para emagrecer [...] (risos) (GN10).

Nas falas acima, foi possível verificar a fragilidade da tolerância social ao excesso de peso em mulheres. O corpo feminino, permeado por valores e significados contraditórios, ora é adorado por seus contornos delgados e sensuais, ora é induzido a se transformar intensamente, aumentar de tamanho e abdicar de si em prol da maternidade.

Como afirma Schwengber, ${ }^{38}$ "a educação dos corpos gravídicos na atualidade reinscreve $o$ corpo da mulher-mãe em um rigoroso regime de vigilância e de regulação, uma tarefa cada vez mais complexa e de muitas aprendizagens e exigências" (p.167).

Sob esta ótica, o gestar com excesso de peso representa um desafio para as mulheres. A amplitude de sentimentos relacionados à percepção das alterações corporais é capaz de despertar tanto a depreciação da autoestima quanto a naturalização do excesso de peso, em um grande jogo de inclusão e exclusão que compromete sua saúde física e emocional. Enquanto gestantes, apesar de perturbador, o simbolismo social da maternidade garante-lhes a proteção. Já no puerpério, os valores estéticos vigentes retomam sua tirania, colocando-lhes em situação de apreensão e vulnerabilidade dupla: conciliar o "reajuste" do corpo obeso à missão de cuidar e nutrir uma nova vida.

Diante disso, ressalta-se a importância do preparo das unidades/equipes de saúde na Atenção Básica para a realização do cuidado durante a gestação e no pós-parto. Garantir um atendimento que proporcione o diagnóstico precoce, tratamento e controle do excesso de peso e o suporte psicológico pela equipe multidisciplinar pode ser um caminho para a prevenção do ganho de peso gestacional excessivo e/ou seu tratamento no puerpério, por meio da construção de vínculos de afetividade e confiança, primordiais para o fortalecimento da mulher. Mas isso só não é 
suficiente, sendo necessário que as equipes multidisciplinares percebam as inúmeras formas de violência simbólica a que estão submetidas as mulheres, para que possam apoiá-las a ocuparem o protagonismo do cuidado de si mesmas.

\section{Considerações finais}

O gestar com excesso de peso foi permeado por expressões das construções sociais sobre o corpo feminino, que geram tensões e conflitos nas mulheres. A ambiguidade entre a naturalização do peso excessivo na condição da mulher-mãe e o sentimento de inadequação ao corpo de mulheresposa socialmente idealizado produz sentimentos de baixa autoestima, limitação na realização de atividades cotidianas e temor em relação à aparência do próprio corpo no puerpério.

Enfim, embora este manuscrito não se proponha a esgotar o assunto sobre os sentidos do ganho de peso excessivo na gestação, espera-se que possa contribuir para o planejamento de políticas e orientação de condutas voltadas a humanização e integralidade das ações de alimentação e nutrição no pré-natal e no puerpério, baseadas no estabelecimento de diálogos horizontais entre profissionais e usuárias e em fluxos de referência para acompanhamento psicológico e nutricional de gestantes assistidas pela Atenção Primária à Saúde do município de Macaé-RJ.

\section{Colaboradoras}

Pires CC e Baião M trabalharam em todas as etapas, desde a concepção do estudo até a revisão da versão final do artigo; Capelli JCS, Rodrigues ML e Santos MMAS participaram da redação, revisão crítica do artigo e da sua versão final.

Conflito de interesses: As autoras declaram não haver conflitos de interesses.

\section{Referências}

1. Brasil. Ministério da Saúde. Secretaria de Atenção à Saúde. Departamento de Atenção Básica. Atenção ao pré-natal de baixo risco. Brasília: Editora do Ministério da Saúde; 2013. 318 p.

2. Melere C, Hoffmann JF, Nunes MAA, Drehmer M, Buss C, Ozcariz SGI, et al. Índice de Alimentação Saudável para Gestantes: adaptação para uso em gestantes brasileiras. Rev Saúde Pública. 2013; 47(1):20-28.

3. Nast M, Oliveira A, Rauber F, Vitolo MR. Ganho de peso excessivo na gestação é fator de risco para o excesso de peso em mulheres. Rev Bras Ginecol Obstet. 2013; 35(12):536-540.

4. World Health Organization. Obesity and overweight [Internet] 16 Feb. 2018. [acesso em: 05 nov. 2017]. Disponível em: http://www.who.int/news-room/fact-sheets/detail/obesity-and-overweight 
5. Brasil. Ministério da Saúde. Secretaria de Vigilância em Saúde. Departamento de Vigilância de Doenças e Agravos não Transmissíveis e Promoção da Saúde. Vigitel Brasil 2016: vigilância de fatores de risco e proteção para doenças crônicas por inquérito telefônico: estimativas sobre frequência e distribuição sociodemográfica de fatores de risco e proteção para doenças crônicas nas capitais dos 26 estados brasileiros e no Distrito Federal em 2016. Brasília: Ministério da Saúde; 2017.160 p.

6. Wanderley EM, Ferreira VA. Obesidade: uma perspectiva plural. Ciênc Saúde Coletiva. 2010; 15(1):185-194.

7. Ferreira VA, Magalhães E. O corpo cúmplice da vida: considerações a partir dos depoimentos de mulheres obesas de uma favela carioca. Ciênc Saúde Coletiva. 2006; 11(2):483-490.

8. Vargas EP. "Barrigão à mostra": vicissitudes e valorização do corpo reprodutivo na construção das imagens da gravidez. Histórias, Ciências, Saúde, Manguinhos. 2012; 19(1):237-258.

9. Barbosa PZ, Rocha-Coutinho ML. Maternidade: novas possibilidades, antigas visões. Rev Psic Clin. 2007; 19(1):163-185.

10. Loth KA, Bauer KW, Wall M, Berge J, Neumark-Sztainer D. Body satisfaction during pregnancy. Body Image. 2011; 8(3):297-300.

11. Sato APS, Fujimori E. Estado nutricional e ganho de peso de gestantes. Rev Latino-Am. Enfermagem. 2012; 20(3):[7 telas].

12. Bardin L. Análise de conteúdo. $4^{a}$ ed. Petrópolis: Vozes; 2009.

13. Instituto Brasileiro de Geografia e Estatística. Estimativas da população residente no Brasil e unidades da federação com data de referência em 10 de julho de 2017 [Internet]. [acesso em: 04 nov. 2017]. Disponível em: ftp://ftp.ibge.gov.br/Estimativas_de_Populacao/Estimativas_2017/estimativa_dou_2017.pdf

14. Macaé. Governo do Estado do Rio de Janeiro. Brasil. [Internet]. [acesso em: 04 nov. 2017]. Disponível em: http://www.macae.rj.gov.br/conteudo/leitura/titulo/infraestrutura-completa

15. Tavares AF, Cautiero GMS, Franco MCV. Relatos e personagens na história de Macaé. Macaé: Solar dos Mellos; 2014.

16. Morse JM. Critical issues in qualitative research methods. Thousand Oaks: Sage; 1994.

17. Brasil. Conselho Nacional de Saúde. Resolução CNS nº66 de 12 de dezembro de 2012. Contém as diretrizes e normas regulamentadoras de pesquisas envolvendo seres humanos. Brasília, DF, 2012b. Disponível em: http://conselho.saude.gov.br/resolucoes/2012/Reso466.pdf

18. Petribu BGC, Mateos MABA. Imagem corporal e gravidez. Junguiana. 2017; 35(1):33-39.

19. Lê Breton D. A sociologia do corpo. 2a ${ }^{\text {a }}$ ed. Petrópolis, RJ: Vozes; 2007.

20. Kanno P, Rabelo M, Melo GF, Giovani A. Discrepâncias na imagem corporal e na dieta de obesos. Rev Nutr. 2008; 21(4):423-430.

21. Serra GMA, Santos EM. Saúde e mídia na construção da obesidade e do corpo perfeito. Ciênc Saúde Coletiva. 2003; 8(3):691-701.

22. Clark A, Skouteris H, Wertheim EH, Paxton SJ, Milgrom J. My baby body: a qualitative insight into women's body-related experiences and mood during pregnancy and the postpartum. J Reprod Infant Psychol. 2009; 27(4):330-345. 
23. Vieira JA. A identidade da mulher na modernidade. Rev Delta. 2005; 21(Especial):207-238.

24. Bourdieu P. A dominação masculina: condição feminina e violência simbólica. Rio de Janeiro: Best Bolso; 2014.

25. Kamysheva E, Skouteris W, Wertheim EH, Paxton SJ, Milgron J. Examination of a multi-factorial model of body-related experiences during pregnancy: the relationships among physical symptoms sleep quality, depression, self-esteem, and negative body attitudes. Body Image. 2008; 5(2):152-163.

26. Araújo NM, Salim NR, Gualda DMR, Silva LCFP. Corpo e sexualidade na gravidez. Rev Esc Enferm USP. 2012; 46(3):552-558.

27. Machado CD, Vinholes DB, Feldens VP. Avaliação da autoestima de gestantes atendidas em um ambulatório no município de Tubarão, Santa Catarina. Arq Catarin Med. 2013; 42(2):50-55.

28. Hauff LE, Demerath EW. Body image concerns and reduced breastfeeding duration in primiparous overweight and obese women. Am J Hum Biol. 2012; 24(3):339-349.

29. Schultheisz TSV, Aprile MR. Autoestima, conceitos correlatos e avaliação. Rev Equilíbrio Corporal Saúde. 2013; 5(1):36-48.

30. Nobre RG, Meireles AVP, Frota JT, Costa RMM, Coutinho VF, Garcia MMCM, et al. Comportamento alimentar e percepção da imagem corporal de gestantes atendidas em um ambulatório de alto risco. Rev Bras Prom Saúde. 2014; 27(2):256-262.

31. Moreira LM. O ganho de peso excessivo no pós-parto na perspectiva das mulheres atendidas na atenção básica de saúde de Manguinhos [Dissertação]. [Rio de Janeiro]: Fundação Osvaldo Cruz, Escola Nacional de Saúde Pública Sérgio Arouca, 2017.

32. Brasil. Ministério da Saúde. Portaria 569 de $1^{\circ}$ de Junho de 2000. Institui o Programa de Humanização no Pré-natal e Nascimento, no âmbito do Sistema Único de Saúde. Diário Oficial da União. 8 jun. 2000; 1:4-6.

33. Meireles JFF, Neves CM, Carvalho PHB, Ferreira MEC. Imagem corporal de gestantes: um estudo longitudinal. J Bras Psiquiatr. 2016; 65(3):223-230.

34. Sui Z, Turnbull D, Dodd J. Effect of body image on gestational weight gain in overweight and obese women. Women Birth. 2013; 26(4):267-272.

35. Sacomori C, Cardoso FL, Vanderlinde C. Pelvic floor muscle strength and body self-perception among Brazilian pregnant women. Physiotherapy. 2010; 96(4):337-343.

36. Skouteris H, Carr R, Wertheim EH, Paxton SJ, Duncombe D. A prospective study of factors that lead to body dissatisfaction during pregnancy. Body Image. 2005; 2(4):47-61.

37. Meireles JFF, Neves CM, Carvalho PHB, Ferreira MEC. Insatisfação corporal em gestantes: uma revisão integrativa da literatura. Ciênc Saúde Coletiva. 2015; 20(7):2091-2103.

38. Schwengber MSV. Mãe moderna: esportiva e forte. Cad Cedes. 2012; 32(87):165-176.

Recebido: 08 de março, 2018

Revisado: 05 de maio, 2018

Aceito: 26 de julho, 2018 